\title{
PPS: Privacy-Preserving Statistics using RFID Tags
}

\author{
Erik-Oliver Blass Kaoutar Elkhiyaoui Refik Molva \\ EURECOM, Sophia Antipolis, France \\ \{blass|elkhiyao|molva\}eeurecom.fr
}

\begin{abstract}
As RFID applications are entering our daily life, many new security and privacy challenges arise. However, current research in RFID security focuses mainly on simple authentication and privacy-preserving identification. In this paper, we widen the scope of RFID security and privacy by introducing a new application scenario. The suggested application consists of computing statistics on private properties of individuals stored in RFID tags. The main idea is to compute global statistics while preserving the privacy of individual readings. PPS assures the privacy of properties stored in each tag through the combination of homomorphic encryption and aggregation at the readers. Re-encryption is used to prevent tracking of users. The readers scan tags and forward the aggregate of their encrypted readings to the back-end server. The back-end server then decrypts the aggregates it receives and updates the global statistics accordingly. Therewith, PPS is provably privacy-preserving. Moreover, tags can be very simple since they are not required to perform any kind of computation, but only to store data.
\end{abstract}

\section{Introduction}

In Radio Frequency IDentification (RFID), tags are transponders that reply to reader queries and send their identifiers. Being cost effective, tags are deployed on a large scale and typically used for identification of goods or even individuals. However, such a deployment comes with new security and privacy threats such as impersonation on the one hand, and tracking of tags and therewith individuals on the other hand. The cost effectiveness also implies strong limitation of computational capabilities of the tags. Current passive RFID tags can hardly afford for security mechanisms relying on complex cryptographic operations to counter the security and privacy threats.

Revisiting security problems such as authentication and privacy preserving identification in the highly constrained setting of RFID tags has given rise to a number of research activities, focusing on lightweight authentication, identification schemes, and formal security and privacy properties thereof, e.g., see Ateniese et al. [1], Bringer and Chabanne [5], Bringer et al. [6], Dimitrou [11], Pietro and Molva [22], Tsudik [26], Vaudenay [27], Weis et al. [28].

In the paper at hand, we introduce a new application scenario, raising new requirements beyond the classical authentication and identification issues. The target scenario is the collection of statistics over private properties of a large population of individuals. Due to RFID users' demands and due to regulatory matters, the main challenge in this scenario is to preserve the privacy of these individuals with respect to their properties.

Addressing this scenario with RFID tags, each tag would contain the attributes of its holder in an encrypted form. The ultimate goal would be to allow a centralized party, 
such as a server, to compute global statistics. For example, the distribution over the properties held by a group of individuals might be of interest, but without disclosing the attributes of individuals to any party involved in the collection of these statistics.

Hence, we suggest a scheme called PPS ("Privacy-Preserving Statistics") that assures privacy of individual attributes in this scenario. In PPS, intermediate parties called readers collect encrypted properties from tags, compute aggregates over encrypted readings without decrypting them, and periodically forward the result of such aggregation operations to the back-end server. The server is then able to compute a global aggregate in cleartext based on the aggregates of the encrypted readings transmitted by readers.

The main challenge in this scenario is to allow the readers to perform the aggregation over encrypted attribute values from which the server can derive global statistics in cleartext. We address this problem through homomorphic encryption. Another threat to the privacy of the tag holders is the tracing of tags by readers. In order to circumvent this threat, we use re-encryption mechanisms. However, the scarcity of resources in tags prohibits the assignment of complex operations to tags. Therefore, the readers will perform re-encryption of the ciphertexts stored on the tags. Thus, tags do not have to perform any cryptographic operations.

The major contributions of PPS are:

- contrary to related work on privacy preserving tag identification, such as Ateniese et al. [1], PPS provides an RFID-based mechanism to collect statistics over a set of properties in a privacy-preserving manner.

- formal proofs of privacy and unlinkability against external eavesdroppers, malicious readers, and curious back-end servers.

- minimal hardware requirements resulting in cheap tags: PPS does not require tags to do any cryptographic computation, tags are passive, i.e., battery-less and only require data storage functions. Contrary to related work, PPS' storage-only requirements enable implementations on today's available EPC class1 Gen2 tags.

- data integrity: tampering with data stored on tags can be detected.

The sequel of this paper is organized as follows. In Section 2, we present a typical scenario for our application, we state the problem, and we derive the requirements for the solution. In Section 4, we present the building blocks of PPS. In Section 3, we define the notion of privacy and unlinkability in the context of our application, and we present the adversary model. Section 5 gives the formal analysis of the protocol. Finally, related work is presented in Section 7.

\section{Problem statement}

In this section, we introduce a typical scenario for PPS, and we present a system model and the requirements PPS should fulfill. 


\subsection{Application scenario}

The solution we propose targets applications involving a central organization that wants to collect statistics on a given population. This population will be equipped with RFID tags that will be read by readers managed by intermediary entities that are independent from the central organization.

We can imagine a scenario where a shopping mall wants to compare the activity of the shops it hosts or which type of clients it attracts the most throughout the year. The shops will deploy readers at the cashiers. The visitors of the shopping mall are provided with RFID tags. To give an incentive to the visitors of shopping malls to carry their tags, we embed RFID tags into loyalty cards that allow tag owners to have discounts or privileged access to parking, restaurants, etc.

Such an incentive requires binding the RFID tag to the identity of the tag owner to ensure that the RFID tag is being used by its owner when scanned. Cheap RFID tags that are the target technology of PPS cannot afford cryptographic authentication. Therefore, we use an out of band authentication such as a printed picture on the loyalty card to verify the identity of the tag holder at payment for instance.

Associating the RFID tag only with the picture of its owner assures that the holder of the tag will be the actual owner while preserving his anonymity with respect to the mall he is visiting.

The RFID tag encodes the private properties of the tag holder, for example gender, age, profession etc. When the tag holder enters a shop for instance, the encrypted properties on the tag will be scanned by a reader. Each reader will aggregate the encrypted data it collects during a period such as a day. At the end of each period, each reader will forward the aggregate data to a server managed by the mall. The server will then update the overall statistics based on the aggregate values sent by the readers.

The key requirement in this scenario is preserving privacy: a solution should allow the server to compute global statistics over private properties of visitors while assuring the privacy of individual properties with respect to the readers and the server.

\subsection{System model}

PPS is the solution we propose to collect privacy preserving statistics. A typical application scenario for PPS involves several parties as follows:

- Issuer $I$ : the issuer initializes each tag by writing into the tag's memory an encrypted representation of the properties of the tag holder.

- Tags $\left\{T_{i}\right\}$ : each tag stores an encrypted representation of the properties of the tag holder. The encrypted representation of $p$ properties in each tag consists of $\left\{P_{i}\right.$, $1 \leq i \leq p\}$ where $P_{i}$ is set to "true" if the tag holder possesses the corresponding property.

- Readers $\left\{R_{i}\right\}$ : readers are in charge of collecting properties stored on tags. They read the data stored on each tag and forward the result of these readings to the back-end server.

- A back-end server $S: S$ processes the aggregate data received from readers and derives some global statistics such as distribution of attendance rate with respect to event types and population characteristics. 
The issuer and the back-end server can be managed by independent parties. For instance, in a typical scenario, social security could act as an issuer, whereas the back-end server would be managed by the shopping mall.

\subsection{Requirements: Privacy \& Unlinkability}

The basic requirement for $S$ is to count the number of tag holders satisfying each property $P_{i}$ for all the properties. The main concern is to gather statistics such as counts about each property $P_{i}$, while preserving the privacy of tag holders. Neither readers nor the back-end server should be able to disclose the values of a tag holder's properties. To ensure privacy in our scheme, we propose a solution that combines encryption and aggregation. In that solution, the list $\omega_{i}$ of the tag holder properties are encrypted as $E\left(\omega_{i}\right)$ and stored on the tag. Through subsequent readings of tags in its range, the reader computes the aggregate of the ciphertexts received from the tags, $\sum E\left(\omega_{i}\right)$, and periodically forwards the encrypted aggregate value to the back-end server as shown in Fig. 1.

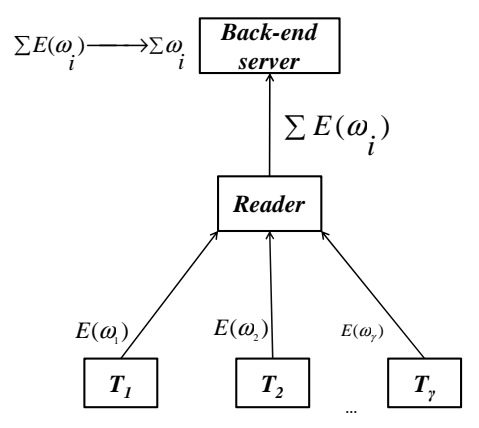

Fig. 1. Aggregation in an RFID-based system

The back-end server $S$ is the only entity that can decrypt ciphertexts. To enforce privacy against $S$, readers must aggregate the ciphertexts received from the tags in their range before forwarding the encrypted data to $S$. If the readers forward data without aggregation to the back-end server $S$, the latter can always tell which properties the tag holders satisfy. Nonetheless, forwarding each individual reading to the server would strongly overload typically embedded, low capacity readers.

Even though the privacy of properties is assured through encryption, unlinkability of tags, as defined by Chatmon et al. [8], has to be assured, too. An adversary should never be able to link two responses of the same tag over different sessions. In order to assure unlinkability, the encrypted property values sent by the same tag should be different for each reading. Re-encryption is used to that effect. In Section 3.2, we formally define the notion of unlinkability. 


\section{Adversary \& Privacy Models}

In this section, we introduce the adversary model and define the notions of privacy and unlinkability for the proposed application.

\subsection{Adversary model}

PPS protects against two different categories of adversaries,

1. $\mathcal{A D} \mathcal{V}_{1}$, external adversaries and malicious readers,

2. $\mathcal{A D} \mathcal{V}_{2}$, a malicious back-end server.

$\mathcal{A D} \mathcal{V}_{1}$ does not collude with $\mathcal{A D} \mathcal{V}_{2}$

$\mathcal{A D \mathcal { V } _ { 1 }}$ : Borrowing notions from Cramer and Damgård [9], we assume a rushing, active adversary who has full control over all communication between tags and readers. He can not only eavesdrop messages, but also intercept, modify, and even initiate communication. For example, the adversary might impersonate a tag and communicate with the reader or read-out tags. He might even replace a tag's content by re-writing it. However, re-writing tags has some special implications on PPS's security, so we discuss this issue separately in Section 6. Finally, the adversary might compromise readers, readout and tamper with their memory and program - consequently, malicious readers might not behave in protocol compliant manner. This adversary corresponds in real world, to a reader that is located near or in the shopping mall for instance. This adversary aims at tracking tag holders and disclosing some of the properties that the tag holders satisfy.

$\mathcal{A D V _ { 2 }}$ : The back-end server might be under the control of the adversary. The backend server is passive in the sense that it only receives aggregates from readers. It cannot initiate communication with tags or readers.

Limitations: We conjecture that there might be scenarios where back-end servers have full control over all communication and might collude with compromised readers, e.g., envisioning an extreme scenario whereby the mall would also control the readers of all shops it hosts. We clearly state that PPS will not provide privacy in such scenarios.

As motivated in the introduction, the adversary's primary goal in any case, i.e., $\mathcal{A D} \mathcal{V}_{1}$ or $\mathcal{A D V _ { 2 }}$, is to gain some knowledge about sensitive information, in this case individual tag holders' properties, as formalized in the following privacy models.

\subsection{Privacy Models}

PPS privacy notions are direct adaptations of privacy definitions originally proposed by Ateniese et al. [1], Golle et al. [15], and experiment-based definitions by Juels and Weis [17].

At the end of a protocol execution, PPS is said to be privacy-preserving, if $\mathcal{A D} \mathcal{V}_{1}$ and $\mathcal{A D} \mathcal{V}_{2}$ can neither decide which properties a given tag satisfies nor link tags to previous protocol executions. In conclusion, an adversary should not have a higher chance 


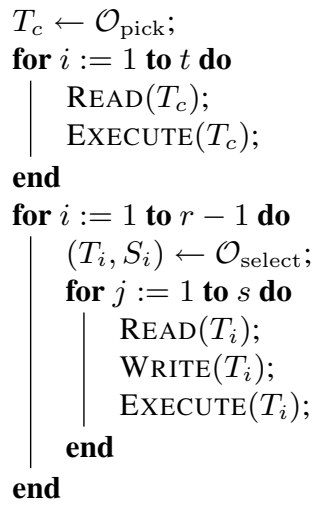

Algorithm 1: Learning phase of $\mathcal{A D} \mathcal{V}_{1}$

$P_{i} \leftarrow$ PICKPROPERTY;

OUPUT $b$;

Algorithm 2: Challenge phase of $\mathcal{A D} \mathcal{V}_{1}$

in breaking privacy or unlinkability than simple guessing. The following oracle-like constructions exist:

$\mathcal{O}_{\text {pick }}$ is an oracle that randomly selects a tag $T_{i}$ from all the $n$ tags in the system.

$\mathcal{O}_{\text {select }}$ is an oracle that randomly returns a tag $T_{i}$ from all the $n$ tags in the system along the list $S_{i}$ of properties $P_{j}$ that tag $T_{i}$ satisfies.

$\mathcal{O}_{\text {flip }}$ is an oracle that, provided with two tags $T_{0}, T_{1}$, randomly chooses $b \in\{0,1\}$ and returns $T_{b}$.

$\mathcal{O}_{\text {aggregate }}$ computes a total of $s$ aggregates $\mathrm{Agg}_{1}, \mathrm{Agg}_{2}, \ldots, \mathrm{Agg}_{s}$, each time by randomly choosing a set of $\gamma$ tags: $\mathrm{Agg}_{1}$ is computed using tags $\left(T_{1}^{1}, T_{1}^{2}, \ldots, T_{1}^{\gamma}\right)$, $\mathrm{Agg}_{2}$ is computed using $\left(T_{2}^{1}, T_{2}^{2}, \ldots, T_{2}^{\gamma}\right), \ldots, \operatorname{Agg}_{s}$ is computed using $\left(T_{s}^{1}, T_{s}^{2}, \ldots, T_{s}^{\gamma}\right)$. The sets of tags are chosen randomly, but there is at least one tag that is an element of two different sets, i.e., used in the computation of two different aggregates. Finally, $\mathcal{O}_{\text {aggregate }}$ returns $\mathrm{Agg}_{1}, \mathrm{Agg}_{2}, \ldots, \mathrm{Agg}_{s}$.

Privacy against $\mathcal{A D} \mathcal{V}_{1}$ An adversary breaks the privacy of PPS, if given a tag $T$ and a property $P_{i}$, he can decide if a tag $T$ satisfies the property $P_{i}$ or not.

To that effect, an adversary $\mathcal{A D} \mathcal{V}_{1}$ has access to tags in two phases. In a learning phase (Algorithm 1), $\mathcal{A D} \mathcal{V}_{1}$ is provided with a challenge tag $T_{c}$ from the oracle $\mathcal{O}_{\text {pick }}$. He can read from $T_{c}$ for a maximum of $t$ times. $\mathcal{O}_{\text {select }}$ gives $r-1$ tags to $\mathcal{A D V} \mathcal{V}_{1}$ along with the list $S_{i}$ of properties $P_{j}$ that each tag $T_{i}$ satisfies. $\mathcal{A D} \mathcal{V}_{1}$ can read and write into $T_{i}$ for a maximum of $s$ times. After each read or write access to a tag in the learning phase, the tag is allowed to interact with a legitimate reader by a normal PPS protocol run, cf., "EXECUTE".

In the second phase, a challenge phase (Algorithm 2), $\mathcal{A D} \mathcal{V}_{1}$ picks a property $P_{i}$ by calling a function PICKPROPERTY. Given the results of the different readings and $T_{c}, \mathcal{A D} \mathcal{V}_{1}$ outputs a bit $b$, such that $b=1$ if he guesses that $T_{c}$ satisfies $P_{i}$, and $b=0$ otherwise.

$\mathcal{A D} \mathcal{V}_{1}$ succeeds, if his guess is right.

Definition 1. PPS is said to be privacy-preserving with respect to $\mathcal{A D} \mathcal{V}_{1}$, if for all adversaries of category $\mathcal{A D} \mathcal{V}_{1}, \operatorname{Pr}\left[\mathcal{A D} \mathcal{V}_{1}\right.$ succeeds $] \leq \frac{1}{2}+\epsilon$, such that $\epsilon$ is negligible. 


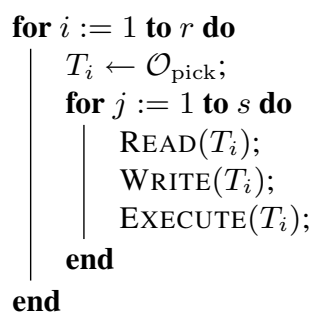

Algorithm 3: Learning phase of $\mathcal{A D V}_{1}$

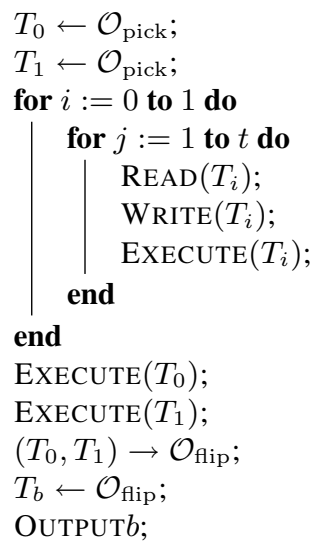

Algorithm 4: Challenge phase of $\mathcal{A D} \mathcal{V}_{1}$

Privacy against $\mathcal{A D} \mathcal{V}_{2}$ As assumed above, $\mathcal{A D} \mathcal{V}_{2}$, i.e., a malicious back-end server, only receives aggregates from readers. In any case, there is no relation between tags, and therewith tag holders, and $\mathcal{A D} \mathcal{V}_{2}$. In conclusion, $\mathcal{A D} \mathcal{V}_{2}$ simply cannot learn anything about properties of tags.

While we do not target a formal proof, privacy against $\mathcal{A D} \mathcal{V}_{2}$ is furthermore discussed and additional reasoning is given in the according security analysis section 5.1.

Unlinkability against $\mathcal{A D} \mathcal{V}_{1}$ The tags targeted in this paper only feature storage capabilities. Hence, tags cannot update the content of their memory themselves after a read and, therefore, the content of a tag's memory does not change between two protocol executions. In the face of an overwhelmingly powerful adversary who can eavesdrop all communications between tags and readers, tags would be trivially linkable. However, we conjecture that it is fair to assume that an adversary in the real world cannot continuously monitor tags and that there is at least one protocol execution that is "un-observed" by the adversary. Once a tag $T$ is re-written outside the range of the adversary, the adversary should not be able to link the previous interactions he has seen to $\operatorname{tag} T$. In accordance with notions of related work such as: insubvertible encryption by Ateniese et al. [1], backward security by Dimitrou [11], and privacy against anonymizers by Sadeghi et al. [23], we assume that there is at least one protocol execution that takes place outside the range of the adversary. Under this assumption, neither external adversaries nor readers should be able to link two responses from the same tag once it is re-written outside their range.

More formally, in a learning phase (Algorithm 3), $\mathcal{A D} \mathcal{V}_{1}$ is provided with $r$ random tags from $\mathcal{O}_{\text {pick. }} \mathcal{A D} \mathcal{V}_{1}$ can read from and write into the $r$ tags for a maximum of $s$ times. After each read or write access, the tags interact with legitimate readers by a PPS execution, cf., "EXECUTE".

In the challenge phase (Algorithm 4$), \mathcal{A D} \mathcal{V}_{1}$ is provided with two challenge tags $T_{0}, T_{1}$ that he is allowed to write into and read from for a maximum of $t$ times. After each access to $T_{0}$ and $T_{1}$ by $\mathcal{A D} \mathcal{V}_{1}, T_{0}$ and $T_{1}$ interacts with a legitimate reader, cf., EXECUTE. 
Then, $T_{0}$ and $T_{1}$ are re-encrypted outside the range of $\mathcal{A D} \mathcal{V}_{1}$ by calling the function EXECUTE. Finally, $\mathcal{O}_{\text {flip }}$ is queried with $T_{0}$ and $T_{1}$ and $\mathcal{O}_{\text {flip }}$ provides $\mathcal{A} \mathcal{D} \mathcal{V}_{1}$ with $T_{b}$. Given the results of the previous readings and $T_{b}$, the adversary $\mathcal{A D} \mathcal{V}_{1}$ guesses the value of $b \in\{0,1\}$. He succeeds, if his guess is right.

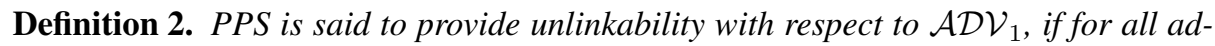
versaries of category $\mathcal{A D} \mathcal{V}_{1}, \operatorname{Pr}\left[\mathcal{A D} \mathcal{V}_{1}\right.$ succeeds $] \leq \frac{1}{2}+\epsilon$, such that $\epsilon$ is negligible.

Unlinkability against $\mathcal{A D} \mathcal{V}_{2}$ An adversary $\mathcal{A D V _ { 2 }}$ should not be able to link aggregates to aggregates it has received before. More precisely, a malicious back-end server should not tell, whether a received aggregate involves a tag that was involved in another aggregate received earlier. $\mathcal{A D} \mathcal{V}_{2}$ has access to the system in two phases. During learning (Algorithm 5), $\mathcal{O}_{\text {aggregate }}$ provides $\mathcal{A D} \mathcal{V}_{2}$ with $s$ aggregates $\mathrm{Agg}_{1}, \ldots, \mathrm{Agg}_{s}$ that he could decrypt.

In the challenge phase (Algorithm 6), $\mathcal{A D} \mathcal{V}_{2}$ outputs a pair $b, b^{\prime} \in\{1, \ldots, s\}$ and therewith $\mathrm{Agg}_{b}$ and $\mathrm{Agg}_{b^{\prime}}$.

$$
\begin{aligned}
& \text { for } i:=1 \text { to } s \text { do } \\
& \left.\right|_{\text {end }} A g g_{i} \leftarrow \mathcal{O}_{\text {aggregate; }}
\end{aligned}
$$

Algorithm 5: Learning phase of $\mathcal{A D V _ { 2 }}$
OutPut $\left(b, b^{\prime}\right)$

Algorithm 6: Challenge phase of $\mathcal{A D V _ { 2 }}$

$\mathcal{A D V _ { 2 }}$ succeeds, if $\mathrm{Agg}_{b}$ and $\mathrm{Agg}_{b^{\prime}}$ have been computed by $\mathcal{O}_{\text {aggregate }}$ with at least one tag in both aggregates.

Definition 3. $P P S$ is said to provide unlinkability with respect to $\mathcal{A D V} \mathcal{V}_{2}$, if for all adversaries of category $\mathcal{A D V}_{2}, \operatorname{Pr}\left[\mathcal{A D V}_{2}\right.$ succeeds $] \leq \frac{1}{s(s-1)}+\epsilon$, such that $\epsilon$ is negligible.

\section{PPS}

Plain encryption of the properties of the tag holders ensures privacy of the data sent to readers. However, encryption prevents aggregation. Conversely, if readers decrypt the data sent by the tag at every reading, the privacy of the tag holder against readers would not be assured. Hence, we suggest to use a standard multiplicative homomorphic encryption scheme, Elgamal [13], in order to allow the aggregation of encrypted data without decryption. Homomorphic encryption allows the back-end server to derive the value $\sum \omega_{i}$ in cleartext from the aggregate of encrypted values $\sum E\left(\omega_{i}\right)$. Furthermore, aggregation is used as privacy enforcement mechanism against the back-end server in order to prevent the back-end server from deriving individual properties of a tag holder.

Even though the privacy of properties is met through homomorphic encryption and aggregation of encrypted readings, these two mechanisms do not ensure the unlinkability of tags. Unlinkability of tags is required in order to prevent the readers or eavesdropping adversaries from tracking tags over different sessions. A basic solution for unlinkability can be provided through re-encryption, cf., Golle et al. [15]. Re-encryption cannot 
be performed by tags, as they are completely passive, therefore, it will be performed by readers. The readers on the other hand, should not be able to decrypt the ciphertexts they receive, otherwise, they can always learn the properties a tag holder satisfies. To tackle this problem, we use an asymmetric encryption that is homomorphic.

As a well studied homomorphic asymmetric encryption scheme, Elgamal [13] meets the requirements of our application, and we use it as the underlying technique. In addition to its homomorphism, Elgamal supports re-encryption. The target scenario for our application calls for an additive homomorphism. However, Elgamal is multiplicatively homomorphic and thus falls short of suiting the target application. To cope with this limitation, the last component of the solution is a special property encoding technique based on Gödel encoding [14]. ${ }^{1}$

\subsection{Elgamal Cryptosystem}

- Setup: the system outputs two large prime $P$ and $Q$ such that $Q$ divides $(P-1)$ and $|P|=\tau$. Here, $\tau$ represents the security parameter of Elgamal. Let $\mathcal{G}$ be a subgroup of $\mathbb{Z}_{\mathrm{P}}^{*}$ of order $Q$, and $g$ be a generator of $\mathcal{G}$. All arithmetic operations will be performed $\bmod P$.

- Key generation: the secret key $s k$ is $x \in \mathbb{Z}_{\mathrm{Q}}$. The public key $p k$ is $y=g^{x}$.

- Encryption: to encrypt a message $\omega \in \mathcal{G}$, one randomly selects $r \in \mathbb{Z}_{\mathrm{Q}}$ and computes $(u, v)=\left(g^{r}, y^{r} \omega\right)$. The ciphertext is $c=(u, v)$.

- Decryption: to decrypt a ciphertext $c=(u, v)$, one computes $\omega=\frac{v}{u^{x}}$.

Elgamal encryption is multiplicatively homomorphic:

$$
\forall \omega_{1}, \omega_{2} \in \mathcal{G}, E\left(\omega_{1}\right) \cdot E\left(\omega_{2}\right)=E\left(\omega_{1} \cdot \omega_{2}\right)
$$

To adapt Elgamal to our scheme, we encode the properties using Gödel encoding before encryption as follows.

\subsection{Gödel Property Encoding}

In order to collect statistics on $p$ properties $P_{i}$, we assign to each property a prime number $p_{i}$. Without loss of generality the first prime number $p_{1}$ will correspond to the property $P_{1}$, the second prime number $p_{2}$ will correspond to $P_{2}$ and so on. Both, properties $P_{i}$ and primes $p_{i}$ are publicly known. If the holder of a tag satisfies two properties $P_{i}, P_{j}$ this will be represented by $\left\{p_{i} p_{j}\right\}$. More formally:

- Setup: let $P_{i}, 1 \leq i \leq p$, be the $p$ properties the back-end server is interested in, and $p_{i}$ are p primes. Each property $P_{i}$ will be mapped to prime number $p_{i}$.

- Encoding: let $m$ be the vector $\left(\nu_{1}, \ldots, \nu_{p}\right)$ such that $\nu_{i}=1$, if the tag $T$ fulfills the property $P_{i}$, otherwise $\nu_{i}=0$. The encoding of the properties of the $\operatorname{tag} T$ is defined as $\Omega(m)=\prod_{i=1}^{p} p_{i}^{\nu_{i}}$.

\footnotetext{
${ }^{1}$ Note that additively homomorphic encryptions such as Paillier [21] or Naccache-Stern [20] may appear to be suitable. However, these schemes do not support an efficient and compact encoding of multiple tag properties, rendering them impractical.
} 


\subsection{Protocol}

In PPS, the tags are initialized once by the issuer. Whenever a tag $T$ is read by a reader $R$, the reader aggregates the ciphertext $c=(u, v)$ it receives from $T$, then it re-encrypts the ciphertext $c$ and writes the new ciphertext into $T$. Periodically, readers in the system forward their aggregates to the back-end server. The latter decrypts and decodes the aggregates and computes the statistics it is interested in.

We assume that the system comprises, for ease of understanding, a single reader, and it has $\gamma$ tags in its range.

- System setup: let $\mathcal{G}$ be a group in which the discrete logarithm is intractable, $g$ a generator of $\mathcal{G}, Q$ the order of $\mathcal{G}$ and $P_{i}$ the $p$ properties of the system. The output of the setup operation is a pair of keys $(p k, s k):\left(y=g^{x}, x\right), x \in \mathbb{Z}_{\mathrm{Q}}$, and $p$ primes $p_{i}$ such that the property $P_{i}$ corresponds to prime number $p_{i}$. Elgamal secret key $s k=x$ is known by both the issuer and the back-end server. Generator $g$, the public key $p k=y$ and the $p$ primes are made public.

- Tag initialization: the input comprises the vector $m=\left(\nu_{1}, \ldots, \nu_{p}\right)$, the public key $y$, the $p$ primes $p_{i}$, and a random number $r \in \mathbb{Z}_{\mathrm{Q}}$. The issuer of the tag encodes the vector $m$ following the Gödel encoding and computes $\omega=\Omega(m)$. The output of the initialization operation is a ciphertext $(u, v)=\left(g^{r}, y^{r} \omega\right)$.

- Aggregation: the input is a set of $\gamma$ ciphertexts $\left(u_{i}, v_{i}\right), 1 \leq i \leq \gamma$, received by the reader from the tags in its range. The reader outputs the aggregate, a new ciphertext $(U, V)=\left(\prod_{i=1}^{\gamma} u_{i}, \prod_{i=1}^{\gamma} v_{i}\right)$, cf., Fig. 2(a).

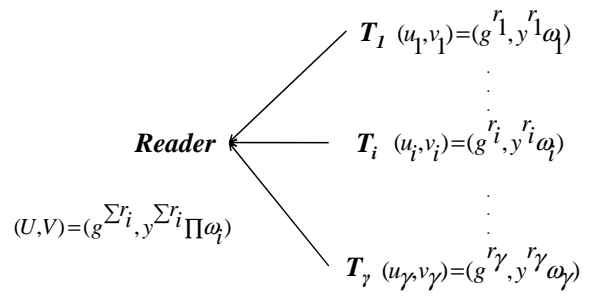

(a) Ciphertext aggregation
Tag

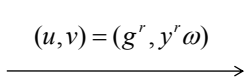

$\left(u^{\prime}, v^{\prime}\right)=\left(g^{r^{\prime}} u, y^{r^{\prime}} v\right)$

(b) Ciphertext re-encryption

Fig. 2. Aggregation and re-encryption in PPS

- Re-encryption: the input of re-encryption is a ciphertext $(u, v)=\left(g^{r}, y^{r} \omega\right)$ received by the reader from a $\operatorname{tag} T, g$ the generator of $\mathcal{G}$, the public key $y$, and a random number $r^{\prime} \in \mathbb{Z}_{\mathrm{Q}}$. The output is a new ciphertext $\left(u^{\prime}, v^{\prime}\right)=\left(g^{\left(r+r^{\prime}\right)}, y^{\left(r+r^{\prime}\right)} \omega\right)$, cf., Fig. 2(b).

On a side note, the value $y^{r} \omega=0 \bmod P$ is considered as "forbidden". When a reader reads a tag that stores 0 , it discards the tag. This means that the reader does not aggregate or re-encrypt the tag, and the reader considers the tag as corrupted. Writing 0 into a tag is a malicious writing attack, see Section 6. 
- Decryption and decoding: the input is a ciphertext $(U, V)=\left(\prod_{i=1}^{\gamma} u_{i}, \prod_{i=1}^{\gamma} v_{i}\right)$ received from the reader, the secret key $x$, and the $p$ primes $p_{i}$. The back-end server computes $W=\frac{V}{U^{x}}$ and factorizes $W$. This factorization is easily feasible, as the back-end server knows the primes $p_{i}$. Given that this factorization is unique, the back-end server gets $\Omega^{-1}(W)=\left(\nu_{1}, \ldots, \nu_{p}\right)$. The respective $\nu_{i}$ corresponds to the number of tags satisfying the property $P_{i}$ that have been read by the reader.

To get the total number of tags satisfying a property $P_{i}$ in the case of multiple readers, the back-end server sums the $\nu_{i}$ for all the readers in the system.

Aggregation under restrictions: In order to ensure the correctness of statistics obtained by the back-end server, we cannot allow the readers to aggregate an infinite number of ciphertexts. They are only allowed to aggregate up to a threshold $\gamma$ of ciphertexts $c_{i}=E\left(\omega_{i}\right)$ at a time, such that $\prod_{i=1}^{\gamma} \omega_{i}<P$.

Evaluation: Typically, $|P|=1024$ bits and $|Q|=160$ bits.

Given $p$ properties $P_{i}$ and $p$ prime numbers $p_{i}$, the threshold $\gamma$ could be defined as $\frac{|P|}{\log _{2}\left(\prod_{i=1}^{p} p_{i}\right)}$. If a reader has $\sigma$ tags in its range, it will aggregate ciphertexts by bunches of size at most $\gamma$. Instead of forwarding one aggregate, the reader forwards $\left\lfloor\frac{\sigma}{\gamma}\right\rfloor+1$ aggregates to the back-end server.

Furthermore, if the readers send to the back-end server the number of tags they read, we can reduce the number of prime numbers used in the Gödel encoding to represent the different properties, cf. Table 1 . This applies in the case we have complementary properties, for instance, $\left(P_{1}, P_{2}\right)=($ male, female $)$. Given the total number of tags read and the number of tag holders satisfying the property $P_{1}$, we deduce the number of tag holders satisfying the property $P_{2}$. Using this fact leads to a more efficient property encoding and thus a larger aggregate size $\gamma$ which improves the privacy of PPS against the back-end server as discussed in Section 5.2.

Table 1. Example properties and their encoding

\begin{tabular}{|l|c|}
\hline Properties & Gödel encoding \\
\hline Male & 2 \\
under 25 & 3 \\
Student & 5 \\
Employee & 7 \\
European union citizen & 11 \\
Disabled & 13 \\
\hline Aggregate size $\gamma$ & 68 \\
\hline
\end{tabular}




\section{Privacy analysis}

This section provides formal proofs for PPS's privacy and unlinkability as defined in the models of Section 3.2.

In this section, we use two additional oracles:

$\mathcal{O}_{\text {semantic }}$ when provided with two plaintexts $\omega_{0}, \omega_{1}$, randomly chooses $b \in\{0,1\}$, encrypts $\omega_{b}$ using Elgamal and public key $p k$, and returns the resulting ciphertext $c_{b}$.

$\mathcal{O}_{\text {semantic-re }}$ when provided with two Elgamal ciphertexts $c_{0}, c_{1}$, randomly chooses $b \in\{0,1\}$, re-encrypts $c_{b}$ using public key $p k$, and returns the resulting ciphertext $c_{b}^{\prime}$.

\subsection{Privacy}

\section{Privacy against $\mathcal{A D} \mathcal{V}_{1}$}

Theorem 1. PPS is privacy-preserving with respect to $\mathcal{A D} \mathcal{V}_{1}$ under the $D D H$ assumption over $\mathcal{G}$.

Proof. Assume we have an adversary $\mathcal{A} \in \mathcal{A D} \mathcal{V}_{1}$ who breaks the privacy experiment. We build an adversary $\mathcal{A}^{\prime}$ that executes $\mathcal{A}$ as a subroutine and breaks the semantic security of Elgamal which leads to a contradiction under DDH. In this proof, we make use of the fact that a $\operatorname{tag} T$ satisfies a property $P_{i}$, iff the corresponding prime number $p_{i}$ divides the plaintext underlying the ciphertext stored on $T$.

- $\mathcal{A}^{\prime}$ picks $p$ properties $P_{i}$ that he maps to $p$ distinct primes $p_{i}$. Then, $\mathcal{A}^{\prime}$ computes $n$ Gödel encodings $\omega_{j}$ using the primes $p_{i}$. Finally, he encrypts $\omega_{j}$ using Elgamal and gets $n$ ciphertexts that he stores on the tags.

- $\mathcal{A}^{\prime}$ specifies two plaintexts $\omega_{0}=\prod p_{i}^{\nu_{0, i}} \leq P-1$ and $\omega_{1}=\prod p_{i}^{\nu_{1, i}} \leq P-1$, such that $\forall i, 1 \leq i \leq p$, and $b^{\prime} \in\{0,1\}: \nu_{b^{\prime}, i} \in\{0,1\}$ and $\nu_{0, i}+\nu_{1, i}=1$. In terms of properties $P_{i}$, this means that tag $T_{0}$, storing plaintext $\omega_{0}$, and tag $T_{1}$, storing $\omega_{1}$, do not have a property in common.

The adversary $\mathcal{A}^{\prime}$ should specify $\omega_{0}$ and $\omega_{1}$ such that $\nu_{0, i}+\nu_{1, i}=1$. Otherwise, $\mathcal{A}$ could choose a challenge property $P_{i}$ that both $\omega_{0}$ and $\omega_{1}$ encode. In this case, the output of $\mathcal{A}$ about $P_{i}$ will not provide the necessary information to $\mathcal{A}^{\prime}$ to break the semantic security of Elgamal. The same holds if $\mathcal{A}$ chooses a property $P_{i}$ that neither $\omega_{0}$ nor $\omega_{1}$ encode.

- $\mathcal{A}^{\prime}$ transmits $\left\{\omega_{0}, \omega_{1}\right\}$ to the oracle $\mathcal{O}_{\text {semantic }}$.

- $\mathcal{O}_{\text {semantic }}$ returns the encryption $c_{b}$ of one of the plaintexts $\omega_{0}, \omega_{1}$ to $\mathcal{A}^{\prime}$.

- $\mathcal{A}^{\prime}$ writes $c_{b}$ into a challenge tag $T_{c}$. Then, $\mathcal{A}^{\prime}$ calls the adversary $\mathcal{A}$ that enters the learning phase. Simulating $\mathcal{O}_{\text {select }}, \mathcal{A}^{\prime}$ provides $\mathcal{A}$ with $r-1$ tags along with the list of properties they are satisfying. $\mathcal{A}$ is allowed to read and write into these tags for a maximum of $s$ times. $\mathcal{A}^{\prime}$ provides $\mathcal{A}$ as well with the challenge tag $T_{\mathrm{c}} . \mathcal{A}$ has only read access to $T_{\mathrm{c}}$ and he is allowed to read it for a maximum of $t$ times. Tags are required to interact with a legitimate reader through the function EXECUTE after being read or written into. As $p k$ is public, $\mathcal{A}^{\prime}$ can simulate successfully EXECUTE.

- $\mathcal{A}$ selects a property $P_{i}$ and outputs 1 , if $T_{c}$ satisfies $P_{i}$ and 0 otherwise.

If $\mathcal{A}$ outputs 1 , this implies that the prime number $p_{i}$ corresponding to $P_{i}$ divides $\omega_{b}$. By construction, $\omega_{0}$ and $\omega_{1}$ do not have any prime divisor in common, and therefore, $\omega_{b}$ is the plaintext dividable by $p_{i}$. 
If $\mathcal{A}$ outputs 0 , this implies that $p_{i}$ does not divide $\omega_{b}$ and by construction $p_{i}$ divides $\omega_{1-b}$. Therefore, $\omega_{b}$ is the plaintext that is not dividable by $p_{i}$.

$\mathcal{A}^{\prime}$ can tell which plaintext $\omega_{b}$ corresponds to $c_{b}$. This breaks the semantic security of Elgamal ensured under the DDH assumption [25], which leads to a contradiction.

Privacy against $\mathcal{A D} \mathcal{V}_{2}$ As stated in Section $3.1, \mathcal{A D} \mathcal{V}_{2}$ receives only aggregated ciphertexts. Still, given the aggregates, $\mathcal{A D} \mathcal{V}_{2}$ can learn some information about the properties of tags read by readers, but is never able to tell which tag, and therewith which holder satisfies which property.

For instance, if $\mathcal{A D V} \mathcal{V}_{2}$ receives an encrypted aggregate from a reader $R$, and decrypts it to Agg $=\prod_{i=1}^{p} p_{i}^{\nu_{i}}$, and $\exists j$ such that $\nu_{j}=0$ after factorization, $\mathcal{A D V} \mathcal{V}_{2}$ can learn that all the tags that were read by $R$ do not satisfy the property $P_{j}$.

However, as $\mathcal{A D} \mathcal{V}_{1}$ and $\mathcal{A D V} \mathcal{V}_{2}$ do not collude, $\mathcal{A D} \mathcal{V}_{2}$ cannot tell which tag satisfies or does not satisfy a certain property $P_{i}$.

\subsection{Unlinkability}

\section{Unlinkability against $\mathcal{A D} \mathcal{V}_{1}$}

Theorem 2. PPS provides tag unlinkability against $\mathcal{A D} \mathcal{V}_{1}$ under the DDH assumption over $\mathcal{G}$.

Proof. The semantic security property of Elgamal encryption can be extended to the semantic security of Elgamal under re-encryption [15]. Let $\mathcal{A}^{\prime}$ be an adversary that chooses two ciphertexts $c_{0}$ and $c_{1}, \mathcal{A}^{\prime}$ then sends $\left\{c_{0}, c_{1}\right\}$ to $\mathcal{O}_{\text {semantic-re. }} \mathcal{O}_{\text {semantic-re }}$ flips a coin $b$, re-encrypts $c_{b}$ to $c_{b}^{\prime}$ and returns $c_{b}^{\prime}$ to $\mathcal{A}^{\prime}$. The semantic security of Elgamal under re-encryption entails that guessing the value of $b$ is as difficult as DDH, see Golle et al. [15].

Now, assume we have an adversary $\mathcal{A} \in \mathcal{A D} \mathcal{V}_{1}$ whose advantage to break the unlinkability experiment is non negligible. We construct a new adversary $\mathcal{A}^{\prime}$ that executes $\mathcal{A}$ and breaks Elgamal's semantic security under re-encryption.

- $\mathcal{A}^{\prime}$ picks $p$ properties $p_{i}, 1 \leq i \leq p$ that he maps to $p$ distinct primes $p_{i}, 1 \leq i \leq$ $p$. Then, he initializes $n$ tags.

- $\mathcal{A}^{\prime}$ calls the adversary $\mathcal{A}$ that enters the learning phase. $\mathcal{A}^{\prime}$ simulates $\mathcal{O}_{\text {pick }}$ and provides $\mathcal{A}$ with $r$ tags. $\mathcal{A}$ is allowed to read and write into these tags for a maximum of $s$ times. After each reading, $\mathcal{A}^{\prime}$ simulates EXECUTE and re-encrypts the ciphertexts, as $p k$ is public.

- $\mathcal{A}$ enters the challenge phase: $\mathcal{A}^{\prime}$ simulates $\mathcal{O}_{\text {pick }}$ and submits tags $T_{0}$ and $T_{1}$ to the adversary $\mathcal{A}$. $\mathcal{A}$ writes into and reads from $T_{0}$ and $T_{1}$ for a maximum of $t$ times. $\mathcal{A}^{\prime}$ can simulate successfully the function EXECUTE as $p k$ is public.

$-\mathcal{A}^{\prime}$ reads the data stored on $T_{0}$ and $T_{1}$. Without loss of generality, let $c_{0}\left(c_{1}\right.$ resp.) denotes the ciphertext stored on $T_{0}$ ( $T_{1}$ resp.). Then, $\mathcal{A}^{\prime}$ transmits $c_{0}$ and $c_{1}$ to the oracle $\mathcal{O}_{\text {semantic-re. }}$.

$-\mathcal{O}_{\text {semantic-re }}$ returns the result $c_{b}^{\prime}$ of re-encrypting one of the two ciphertexts to $\mathcal{A}^{\prime}$. $\mathcal{A}^{\prime}$ writes $c_{b}^{\prime}$ into a tag $T$. 
- $\mathcal{A}^{\prime}$ calls $\mathcal{A}$ and provides him with $T$, simulating $\mathcal{O}_{\text {flip }}$. Then, $\mathcal{A}$ outputs his guess for the value of $b$.

Since $\mathcal{A}$ 's advantage in the unlinkability experiment is non negligible, $\mathcal{A}$ can tell which tag corresponds to the new ciphertext $c_{b}^{\prime}$. If $\mathcal{A}$ outputs 0 , this means that $c_{b}^{\prime}$ is re-encryption of $c_{0}$, otherwise $c_{b}^{\prime}$ is a re-encryption of $c_{1}$. Therefore, $\mathcal{A}^{\prime}$ can break the semantic security under re-encryption of Elgamal that is ensured under the DDH assumption [15], again leading to a contradiction.

\section{Unlinkability against $\mathcal{A D} \mathcal{V}_{2}$}

Theorem 3. PPS provides unlinkability of tags against $\mathcal{A D} \mathcal{V}_{2}$ for large $\gamma$.

Proof (Sketch). An aggregate Agg $=\prod_{i=1}^{p} p_{i}^{\nu_{i}}$ is called completely blinded, iff $\forall i$, $1 \leq i \leq p: \nu_{i}>0$. Now, given a sufficiently large $\gamma$, the aggregates received by the back-end server will be completely blinded with high probability.

Therefore, the back-end server cannot distinguish between the tags involved in the aggregates. Moreover, using a large $s$ in the learning phase would not give the adversary $\mathcal{A D} \mathcal{V}_{2}$ a greater advantage in guessing $\left(b, b^{\prime}\right)$.

In the following, we compute an upper bound of the advantage $\epsilon$ of $\mathcal{A D V _ { 2 }}$ in the unlinkability experiment.

Let $E$ be the event that aggregate Agg is completely blinded, so $\forall i, 1 \leq i \leq p$ : $\nu_{i}>0$. Let $\gamma$ be the number of ciphertexts participating in the aggregate, and $\pi_{i}$ is the probability that a tag holder satisfies property $P_{i}$. Without loss of generality, we assume $\pi_{1} \leq \pi_{2} \leq \ldots \leq \pi_{p}$. Then, the probability that $\nu_{i}=0$ is $\operatorname{Pr}\left(\nu_{i}=0\right)=\left(1-\pi_{i}\right)^{\gamma} \leq$ $\left(1-\pi_{1}\right)^{\gamma}$.

Let $\bar{E}$ be the complementary event of $E$. Therefore, $\operatorname{Pr}(\bar{E})=\operatorname{Pr}\left(\nu_{1}=0 \vee \nu_{2}=\right.$ $\left.0 \ldots \vee \nu_{p}=0\right)$

$\operatorname{Pr}(\bar{E}) \leq \sum_{i=1}^{p} \operatorname{Pr}\left(\nu_{i}=0\right) \leq p\left(1-\pi_{1}\right)^{\gamma}$.

$\epsilon=\operatorname{Pr}(\bar{E})$ is the advantage of $\mathcal{A D} \mathcal{V}_{2}$ in the unlinkability experiment which is negligible in $\gamma$. Therefore, we say that PPS is $\epsilon$-unlinkable against $\mathcal{A D} \mathcal{V}_{2}$, such that $\epsilon \leq p\left(1-\pi_{1}\right)^{\gamma}$.

Note that the advantage of $\mathcal{A D} \mathcal{V}_{2}$ heavily depends on the probability $\pi_{1}$. If $\pi_{1}$ is very small, i.e., representing a rare property such as being disabled, PPS cannot provide unlinkability against $\mathcal{A D} \mathcal{V}_{2}$. In such a case, the back-end server can link tags to aggregates. For instance, if the back-end server sees two aggregates where the property "disabled" is satisfied, it can guess with a non negligible probability that these two aggregates have one tag in common.

\section{Security Analysis}

Tags in our scheme have a writable memory, where the ciphertext is stored every time it is re-encrypted by readers. As there is no access control on tags to check the authenticity of readers, our scheme is vulnerable to "malicious writing".

We can divide malicious writing attacks into two categories: 
- Writing an invalid ciphertext ("garbage") into the tag: this attack can be detected at the back-end server, as decryption and Gödel decoding will not succeed. Moreover, if the adversary writes the value 0 into the tag, this will be detected at the next honest reader to read the tag.

- Writing a valid ciphertext into the tag: a malicious reader could try to alter statistics. The simplest way to implement such an attack is by copying the content of a tag into another one ("cloning"). Moreover, given that Elgamal is malleable and that the adversary knows the primes, the adversary can generate a set of valid ciphertexts from a ciphertext he has seen [12]. Since the ciphertext written into the tag is a valid one, this type of attack cannot directly be detected at decryption, and we will tackle it in the following.

Malicious writing affects the correctness of the results obtained at the back-end server. Given that access control is not feasible in our read-write only tags, this attack cannot be prevented. However, we propose the following solution to detect ciphertexts written by an adversary at the back-end server.

Instead of one ciphertext, each tag stores two ciphertexts $\left(c, c_{I D}\right)$. The first ciphertext $c$ encrypts the properties of the tag holder as described in the previous section. The second ciphertext $c_{I D}$ encrypts a unique ID of the tag using standard Elgamal encryption. After a tag is scanned by a reader, the reader re-encrypts both ciphertexts $c$ and $c_{I D}$ and writes the new ciphertexts into the tag. The reader aggregates $c$ and keeps a record of $c_{I D}$. During decryption at the back-end server, if the back-end server suspects that a received aggregate is not correct, he contacts a "trusted third party". This trusted third party (TTP) checks the records $c_{I D}$ stored at the readers. TTP decrypts these ciphertexts and gets the IDs of the tags that were scanned along with the corresponding properties of their holders. In this manner, the TTP detects tag cloning as the ID of the cloned tag will be repeated several times.

Furthermore, in order to detect tag tampering, the tag issuer should keep a database of the tag IDs and their corresponding properties and reveal it to the TTP. Therewith, the TTP can compare the decrypted properties and the actual properties stored in the issuer database. If there is a discrepancy between the properties corresponding to the same tag ID, the TTP reports a fraud. Meanwhile, the TTP does not reveal the records of the IDs stored on the readers either to the back-end server or to the readers.

We clearly acknowledge that readers can fake statistics. However, the readers could be provided with an incentive to encourage them to ensure the integrity of the results obtained at the back-end server and therefore not to tamper with tags' content or fake statistics. For instance, the statistics could be used by the shops to define their marketing strategy. As mentioned before, PPS focuses on privacy and unlinkability of tag holders.

\section{Related work}

Juels et al. [18] utilize re-encryption to protect privacy of RFID-enabled banknotes. Each time a banknote is spent, the readers in shops or banks re-encrypt the encrypted serial number of the banknote stored on the tag. The main drawback of this scheme is that the authorized readers have access to the plaintext underlying the ciphertext spoiling unlinkability. Similarly, Golle et al. [15] introduce universal re-encryption allowing 
special re-encryption without knowing the public key initially used to encrypt the plaintexts. While this protocol provides key privacy, it fails at providing unlinkability after malicious writing. An adversary can write his own message $m$ into a tag and encrypt it under its public key. Therewith, the adversary can always link the tag.

Ateniese et al. [1] tackle the above problems by proposing insubvertible encryption, i.e., universal re-encryption and randomized certificates. If the certificate is valid, the ciphertext stored on the tag will be re-encrypted. Otherwise, it will be replaced by a dummy encryption. Ateniese et al. [1] aim at privacy preserving identification, but not privacy preserving statistics collection which is the focus of PPS. Also, Ateniese et al. [1], as well as the results presented by Blundo et al. [4], require special message encodings to map messages to points on elliptic curves. However, currently known efficient encoding schemes fail at preserving the homomorphic properties that are the essential prerequisite for PPS.

Camenisch and Groß [7] propose an attribute encoding for anonymous credentials. The scheme allows users to prove the possession of an attribute with a given value while preserving the privacy of the users. While such an approach could be used to "emulate" privacy-preserving computation of statistics, the main drawback is the requirement for complex interactive proofs between tags and readers- infeasible in our setting with storage-only tags.

Han et al. [16] present a protocol to estimate the total number of tags in the vicinity of a reader. The main idea is to infer this number by examining the number of empty and collision slots in the framed slotted Aloha protocol used for communication. Although [16] enables estimating the total number of tags anonymously, it does not lend itself to collect statistics on tag properties as targeted in the paper at hand.

Kerschbaum et al. [19] propose to privately compute performance properties of an RFID supply chain using data stored on tags. However, this work focuses on computing these metrics without leaking sensitive information of the supply-chain's parties. Kerschbaum et al. [19] use additive homomorphic encryption that does not support collecting statistics on multiple properties and consequently cannot be as efficient as PPS.

Relation to voting: Note that, at first glance, PPS appears to be similar to voting, cf., Benaloh and Tuinstra [3], Sako and Kilian [24]. Instead of counting the number of tag holders satisfying a property $P_{i}$, we count the number of voters voted for a given candidate. However, the RFID settings considered in this paper are very constrained regarding a secure voting application. In secure voting, the voters are required to perform additional complex operations such as public key encryption and zero knowledge proofs to ensure not only privacy and correctness of the votes, but also other sophisticated properties such as receipt freeness, and universal verifiability, cf., Baudron et al. [2], Benaloh and Tuinstra [3], Cramer et al. [10], Sako and Kilian [24]. Clearly, these operations cannot be performed by read/write only tags.

\section{Conclusion}

RFID systems can be used for many applications besides identification and authentication. In this paper, we introduced a new application for RFID that collects statistics over a population of tag holders. We presented PPS, a protocol to mitigate resulting new pri- 
vacy problems. PPS does not require tags to perform any (cryptographic) computation. Instead, tags only need to feature some cheap storage. All computations within PPS are solely performed by readers. PPS provably ensures the privacy of tags and therewith holders' properties as well as their unlinkability: tag holders can be sure that neither RFID readers, nor a back-end system can reveal the properties stored on their tags. Additionally, if scanned at different readers on different occasions, tag holders can be sure that these occasions cannot be linked.

\section{References}

[1] Giuseppe Ateniese, Jan Camenisch, and Breno de Medeiros. Untraceable rfid tags via insubvertible encryption. In CCS '05: Proceedings of the 12th ACM conference on Computer and communications security, pages 92-101, New York, NY, USA, 2005. ACM. ISBN 1-59593-226-7.

[2] Olivier Baudron, Pierre-Alain Fouque, David Pointcheval, Jacques Stern, and Guillaume Poupard. Practical multi-candidate election system. In PODC '01: Proceedings of the twentieth annual ACM symposium on Principles of distributed computing, pages 274-283, New York, NY, USA, 2001. ACM. ISBN 1-58113-383-9.

[3] Josh Benaloh and Dwight Tuinstra. Receipt-free secret-ballot elections (extended abstract). In STOC '94: Proceedings of the twenty-sixth annual ACM symposium on Theory of computing, pages 544-553, New York, NY, USA, 1994. ACM. ISBN 0-89791-663-8.

[4] Carlo Blundo, Angelo De Caro, and Giuseppe Persiano. Untraceable tags based on mild assumptions. Cryptology ePrint Archive, Report 2009/380, 2009. http://eprint. iacr.org/.

[5] Julien Bringer and Hervé Chabanne. Trusted-HB: A low-cost version of $\mathrm{HB}^{+}$secure against man-in-the-middle attacks. IEEE Transactions on Information Theory, 54(9):4339_ 4342, 2008. ISSN 0018-9448.

[6] Julien Bringer, Hervé Chabanne, and Emmanuelle Dottax. $\mathrm{HB}^{++}$: a lightweight authentication protocol secure against some attacks. In SecPerU, pages 28-33, 2006. ISBN 0-76952549-0.

[7] Jan Camenisch and Thomas Groß. Efficient attributes for anonymous credentials. In CCS '08: Proceedings of the 15th ACM conference on Computer and communications security, pages 345-356, New York, NY, USA, 2008. ACM. ISBN 978-1-59593-810-7.

[8] Christy Chatmon, Tri van Le, and Mike Burmester. Secure anonymous RFID authentication protcols. Technical report, Florida State University, Department of Computer Science, Tallahassee, Florida, USA, 2006. http://www.cs.fsu.edu/ burmeste/ TR-060112.pdf.

[9] Ronald Cramer and Ivan Damgård. Introduction to secure multi-party computations. In Contemporary Cryptology: Advanced Courses in Mathematics, pages 41-87. Birkhauser, 2005. ISBN 3-7643-7294-X.

[10] Ronald Cramer, Rosario Gennaro, and Berry Schoenmakers. A secure and optimally efficient multi-authority election scheme. In Advances in Cryptology - EUROCRYPT '97, Lecture notes in computer science, pages 103-118. Springer-Verlag, 1997. ISBN 978-3540-62975-7.

[11] Tasso Dimitrou. rfidDOT: RFID delegation and ownership transfer made simple. In Proceedings of International Conference on Security and privacy in Communication Networks, Istanbul, Turkey, 2008. ISBN 978-1-60558-241-2.

[12] Danny Dolev, Cynthia Dwork, and Moni Naor. Non-malleable cryptography. In STOC '91: Proceedings of the twenty-third annual ACM symposium on Theory of computing, pages 542-552, New York, NY, USA, 1991. ACM. ISBN 0-89791-397-3.

[13] Taher El Gamal. A public key cryptosystem and a signature scheme based on discrete logarithms. In Proceedings of CRYPTO 84 on Advances in cryptology, pages 10-18, New York, NY, USA, 1985. Springer-Verlag New York, Inc. ISBN 0-387-15658-5. 
[14] Kurt Gödel. über formal unentscheidbare sätze der principia mathematica und verwandter systeme i. Monatsheft für Mathematik und Physik, 38:173-198, 1931. ISSN 0026-9255.

[15] Philippe Golle, Markus Jakobsson, Ari Juels, and Paul Syverson. Universal re-encryption for mixnets. In In Proceedings of the 2004 RSA Conference, Cryptographer's track, pages 163-178. Springer-Verlag, 2002.

[16] Hao Han, Bo Sheng, Chiu C. Tan, Qun Li, Weizhen Mao, and Sanglu Lu. Counting RFID tags efficiently and anonymously. In Proceeding of IEEE Infocom, San Diego, USA, 32010.

[17] Ari Juels and Stephen A. Weis. Defining strong privacy for RFID. In PERCOMW '07: Proceedings of the Fifth IEEE International Conference on Pervasive Computing and Communications Workshops, pages 342-347, Washington, DC, USA, 2007. IEEE Computer Society. ISBN 0-7695-2788-4.

[18] Ari Juels, Ravikanth Pappu, and Thingmagic Llc. Squealing euros: Privacy protection in rfid-enabled banknotes. In Financial Cryptography'03, pages 103-121. Springer-Verlag, 2002. ISBN 978-3-540-40663-1.

[19] F. Kerschbaum, N. Oertel, and L. Weiss Ferreira Chaves. Privacy-preserving computation of benchmarks on item-level data using rfid. In 3rd ACM conference on Wireless network security, pages 105-110, New York, USA, 2010.

[20] D. Naccache and J. Stern. A new public key cryptosystem based on higher residues. In ACM conference on Computer and communications security, pages 59-66, New York, 1998.

[21] Pascal Paillier. Public-key cryptosystems based on composite degree residuosity classes. In Advances in Cryptology EUROCRYPT'99, pages 223-238. Springer-Verlag, 1999. ISBN 3-540-65889-0.

[22] Roberto Di Pietro and Refik Molva. Information confinement, privacy, and security in RFID systems. In Lecture Notes in Computer Science, Volume 4734, pages 187-202, 2007. ISBN 978-3-540-74834-2.

[23] Ahmad-Reza Sadeghi, Ivan Visconti, and Christian Wachsmann. Anonymizer-Enabled Security and Privacy for RFID. In 8th International Conference on Cryptology And Network Security - CANS'09, Kanazawa, Ishikawa, Japan, December 2009. Springer. ISBN 978-3642-10432-9.

[24] Kazue Sako and Joe Kilian. Secure voting using partially compatible homomorphisms. In CRYPTO '94: Proceedings of the 14th Annual International Cryptology Conference on Advances in Cryptology, pages 411-424, London, UK, 1994. Springer-Verlag. ISBN 3540-58333-5.

[25] Yiannis Tsiounis and Moti Yung. On the security of elgamal based encryption. In $P K C^{\prime} 98$, LNCS 1431, pages 117-134. Springer-Verlag, 1998. ISBN 978-3-540-64693-8.

[26] Gene Tsudik. YA-TRAP: yet another trivial RFID authentication protocol. In Proceedings of International Conference on Pervasive Computing and Communications Workshops, Pisa, Italy, 2006. ISBN 0-7695-2520-2.

[27] Serge Vaudenay. On privacy models for RFID. In Advances in Cryptology - Asiacrypt 2007, Lecture Notes in Computer Science, pages 68-87. Springer-Verlag, 2007. ISBN 978-3-54076899-9.

[28] S.A. Weis, S.E. Sarma, R.L. Rivest, and D.W. Engels. Security and privacy aspects of low-cost radio frequency identification systems. In Security in Pervasive Computing, pages 201-212, Boppard, Germany, 2003. ISBN 3-540-20887-9. 